\title{
Effect of Prostaglandin Analogues on Central Corneal Thickness: 3-Year Follow-up Results
}

\author{
Minsu Jang, Kyung Eun Kang, Byung Joo Cho \\ Department of Ophthalmology, Konkuk University Medical Center, Konkuk University School of Medicine, Seoul, Korea
}

Purpose: To evaluate the effects of each subgroup of prostaglandin analogues (PGAs) on central corneal thickness (CCT) in patients with normal tension glaucoma (NTG).

Methods: We retrospectively reviewed 55 eyes of 55 patients with NTG who were receiving PGA therapy. Patients who were treated with $0.005 \%$ latanoprost (16 eyes), $0.0015 \%$ tafluprost (16 eyes), or $0.004 \%$ travoprost (23 eyes) monotherapy were included. CCT assessments were performed at baseline and 1, 2, and 3 years after initiation of treatment.

Results: In the NTG group, the mean CCT showed a decreasing trend, and there was a significant difference in mean CCT at 1 , 2 , and 3 years compared with baseline (baseline, 538.16 $\pm 32.14 ; 1$ year, $526.55 \pm 37.30 \mu \mathrm{m}[p=0.00] ; 2$ years, $521.67 \pm 36.79$ $\mu \mathrm{m}[p=0.00] ; 3$ years, $520.43 \pm 36.88 \mu \mathrm{m}[p=0.00])$. The reduction of CCT was confirmed by subgroup analysis. In the $0.005 \%$ latanoprost group, mean CCT was decreased at 1 year $(p=0.11)$, 2 years $(p=0.00)$, and 3 years $(p=0.02)$. In the $0.0015 \%$ tafluprost group and the $0.004 \%$ travoprost group, mean CCT was also significantly decreased at all years $(p=0.00)$. No statistical difference was observed between the NTG subgroups $(p=0.06)$.

Conclusions: Topical therapy with PGAs appeared to cause a significant decrease in CCT reduction in patients with NTG. A long-term follow-up study including more participants is needed.

Key Words: Central corneal thickness, Low tension glaucoma, Prostaglandin analogue

Normal tension glaucoma (NTG) is a chronic progressive optic neuropathy and is the second most prevalent cause of blindness and irreversible vision deterioration [13]. In east Asia, NTG is the most common form of primary open angle glaucoma, accounting for $77 \%$ of cases in Korea $[3,4]$.

Despite many controversies regarding the origin and

Received: October 22, 2019 Final revision: February 13, 2020 Accepted: May 7, 2020

Corresponding Author: Byung Joo Cho, MD, PhD. Department of Ophthalmology, Konkuk University Medical Center, 120-1 Neungdong-ro, Gwangjin-gu, Seoul 05029, Korea. Tel: 82-2-2030-7651, Fax: 82-2-20305273, E-mail: bjcho@kuh.ac.kr pathogenesis of NTG, major current treatment modalities are directed toward reduction in intraocular pressure (IOP) [5]. Topical prostaglandin analogues (PGAs) are generally the first-line drugs in glaucoma therapy. They have been widely used because of their efficacy, low systemic side effects, and convenience of only once daily use [6]. Although a clear mechanism has not been elucidated, it has been suggested that PGAs are associated with IOP reduction through aqueous humor outflow enhancement within the uveoscleral outflow pathway, accompanied by collagen degradation in the ciliary body $[7,8]$. The ciliary body contains several prostaglandin receptors, whose activation seems to be mediated by matrix metalloproteinase (MMP) synthesis, which is involved in extracellular matrix remod- 
eling $[7,8]$. Considering that the cornea is mainly composed of collagen fibers, it can be assumed that topical PGAs affect central corneal thickness (CCT) [9]. There are many topical PGAs currently in use, including $0.005 \%$ latanoprost, $0.0015 \%$ tafluprost, and $0.004 \%$ travoprost.

Accurate IOP measurement is important for proper treatment of NTG patients. The gold standard for IOP measurement is Goldmann applanation tonometry, but its results may be affected by CCT $[10,11]$. Furthermore, CCT has been suggested to be an independent risk factor for progression in NTG patients $[12,13]$. Therefore, it is important to determine CCT values serially during follow-up for glaucoma, especially in patients treated with PGAs.

Several studies have reported a reduction in CCT after PGA treatment in NTG patients [5,14-16]. However, few studies have compared the change in CCT values associated with each PGA monotherapy in patients with NTG.

In this study, we evaluated the effects of each PGA subgroup on CCT in NTG patients over a 3-year timespan.

\section{Materials and Methods}

\section{Study population}

We performed a retrospective study on data from patients examined at the Department of Ophthalmology at Konkuk University Medical Center from January 1, 2010, to January 31, 2017. A total of 55 eyes from 55 NTG patients were enrolled in the NTG group, and 105 eyes from 105 untreated suspected glaucoma patients were enrolled in the control group. The inclusion criteria for the NTG group were newly diagnosed bilateral NTG treated with 0.005\% latanoprost (Xalatan; Pfizer, New York, NY, USA), $0.0015 \%$ tafluprost (Taflotan; Santen Oy, Tampere, Finland), or $0.004 \%$ travoprost (Travatan; Alcon Laboratories, Fort Worth, TX, USA) as the first-line monotherapy for more than 3 years without change in regimen. NTG was defined by open-angle and confirmed by gonioscopy as follows: repeated measurements of untreated IOP $\leq 21$ $\mathrm{mmHg}$ and a typical glaucomatous optic nerve change corresponding to a visual field defect. The inclusion criteria for the control group were patients with suspected glaucoma, suspicious discs with normal visual field, and IOP $\leq 21 \mathrm{mmHg}$. Patients with other ocular diseases, those who had undergone refractive surgery or intraocular surgery, those with corneal abnormalities, those with other fundus changes preventing reliable optic disc evaluation, or those with other neurologic disorders that could affect the optic nerve were excluded from this study.

This study followed the tenets of the Declaration of Helsinki and was approved by the local institutional review board/ethics committee at Konkuk University Medical Center (KUH1100064). Informed consent was waived due to the retrospective nature of the study.

\section{CCT and IOP}

The CCT was measured with an ultrasound pachymeter (AL- 2000; Bio \& Pachymeter; Tomey, Nagoya, Japan) with patients in the upright position; patients were touched lightly at the center of the cornea and perpendicular to the corneal surface by the same physician. IOP was also measured in both eyes by Goldmann applanation tonometry with proparacaine anesthesia. Results are the mean of five consecutive readings. CCT and IOP measurements were detected before treatment and after 1, 2, and 3 years of treatment.

\section{Statistical analyses}

Statistical analyses were performed using PASW Statistics ver. 18.0 for windows (SPSS Inc., Chicago, IL, USA). Pearson's chi-square test was performed to compare the ratio of sex. An independent sample $t$-test was used to compare age, baseline IOP, and baseline CCT between control and NTG groups. A paired-sample $t$-test was used to evaluate CCT change at each time point. One-way analysis of variance with Bonferroni post-hoc test was performed to evaluate the differences of age and CCT between PGA subgroups. For comparison between groups, the analysis of covariance test was performed, with basal pachymetry as the cofactor. A $p$-value $<0.05$ was considered statistically significant.

\section{Results}

Demographic data at baseline are summarized in Table 1 and 2. Patients who were treated with $0.005 \%$ latanoprost (16 eyes), $0.0015 \%$ tafluprost (16 eyes), or $0.004 \%$ travoprost (23 eyes) monotherapy were included. A total of 105 eyes 
Table 1. Baseline characteristics

\begin{tabular}{lccc}
\hline & NTG group & Control group & $p$-value \\
\hline No. of patients (eyes) & $55(55)$ & $105(105)$ & - \\
Male : female & $29: 26$ & $47: 58$ & $0.10^{*}$ \\
Age (yr) & $56.86 \pm 14.00$ & $54.13 \pm 12.59$ & $0.21^{\dagger}$ \\
Mean baseline IOP $(\mathrm{mmHg})$ & $15.29 \pm 2.75$ & $15.69 \pm 2.98$ & $0.42^{\dagger}$ \\
Mean baseline CCT $(\mu \mathrm{m})$ & $538.16 \pm 32.14$ & $553.38 \pm 30.80$ & $0.00^{\dagger}$ \\
\hline
\end{tabular}

Values are presented as number or mean \pm standard deviation.

$\mathrm{NTG}=$ normal tension glaucoma; $\mathrm{IOP}=$ intraocular pressure; $\mathrm{CCT}=$ central corneal thickness .

*Pearson's chi-square; 'Independent sample $t$-test.

Table 2. Baseline characteristics of each normal tension glaucoma subgroup

\begin{tabular}{|c|c|c|c|c|}
\hline & $\begin{array}{c}0.005 \% \\
\text { latanoprost }\end{array}$ & $\begin{array}{l}0.0015 \% \\
\text { tafluprost }\end{array}$ & $\begin{array}{l}0.004 \% \\
\text { travoprost }\end{array}$ & $p$-value \\
\hline No. of patients (eyes) & $16(16)$ & $16(16)$ & $23(23)$ & - \\
\hline Age (yr) & $53.19 \pm 15.81$ & $57.13 \pm 12.49$ & $59.22 \pm 13.72$ & $0.42^{*}$ \\
\hline Mean baseline IOP $(\mathrm{mmHg})$ & $17.13 \pm 3.10$ & $14.81 \pm 2.17$ & $14.35 \pm 2.31$ & $0.00^{*}$ \\
\hline Mean baseline CCT $(\mu \mathrm{m})$ & $549.38 \pm 40.02$ & $534.31 \pm 26.85$ & $533.04 \pm 28.68$ & $0.26^{*}$ \\
\hline
\end{tabular}

Values are presented as number or mean \pm standard deviation. $\mathrm{IOP}=$ intraocular pressure; $\mathrm{CCT}=$ central corneal thickness. * One-way analysis of variance with Bonferroni post hoc test.

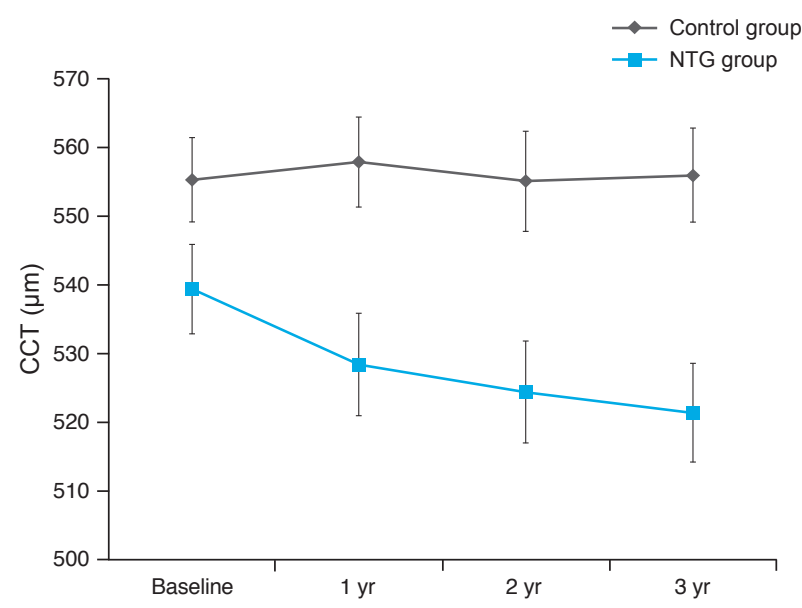

Fig. 1. Mean central corneal thickness (CCT) at baseline and follow-up in control and normal tension glaucoma (NTG) groups. In the control group, the mean CCT is as follows: baseline, $553.38 \pm$ $30.80 \mu \mathrm{m}$; 1 year, $556.49 \pm 31.18 \mu \mathrm{m}(p=0.06) ; 2$ years, $553.94 \pm$ $35.95 \mu \mathrm{m}(p=0.81)$; 3 years, $554.11 \pm 32.49 \mu \mathrm{m}(p=0.68)$. In the NTG group, the mean CCT is as follows: baseline, $538.16 \pm 32.14$ $\mu \mathrm{m}$; 1 year, $526.55 \pm 37.30 \mu \mathrm{m}(p=0.00) ; 2$ years, $521.67 \pm 36.79$ $\mu \mathrm{m}(p=0.00) ; 3$ years, $520.42 \pm 36.88 \mu \mathrm{m}(p=0.00)$. A $p$-value is obtained by comparing each time period with baseline. of 105 controls with suspected glaucoma was included. There were no significant differences in sex $(p=0.10)$, age $(p=0.21)$, and baseline IOP $(p=0.42)$ between the control and NTG groups, but there was a significant difference in CCT $(p=0.00)$ (Table 1). There were significant differences in baseline IOP $(p=0.00)$ but no significance in CCT ( $p$ $=0.26$ ) between the NTG subgroups (Table 2).

There was no statistically significant change in CCT value compared with baseline in the control group. In the NTG subgroup, mean CCT showed a decreasing trend, and there was a significant difference in mean CCT at 1, 2, and 3 years compared with baseline $(p=0.00)$, except for CCT at 1 year for latanoprost ( $p=0.11)$ (Fig. 1).

The reduction of CCT was confirmed by sub-analysis of each NTG subgroup. In the $0.005 \%$ latanoprost group, $0.0015 \%$ tafluprost group, and $0.004 \%$ travoprost group, the mean CCT was significantly decreased at all years (Fig. 2). No statistical difference was observed among the NTG subgroups $(p=0.18)$ (Table 3$)$. 


\section{Discussion}

PGAs are a first-line topical drug for NTG patients due to their ability to reduce IOP, show high efficacy and minimal side effects, and once-daily dosing [17,18]. Although the mechanism for this PGA reduction of IOP is not fully understood, it has been proposed that they act primarily through upregulation of MMPs. MMPs degrade collagen, a basement membrane constituent, and other components in the extracellular matrix. MMPs are found in the corneal epithelium, stroma, endothelium, aqueous humor, and trabecular meshwork $[7,8,19]$. The predominant IOP effect of PGAs is known to be caused by MMP-1 activation in the

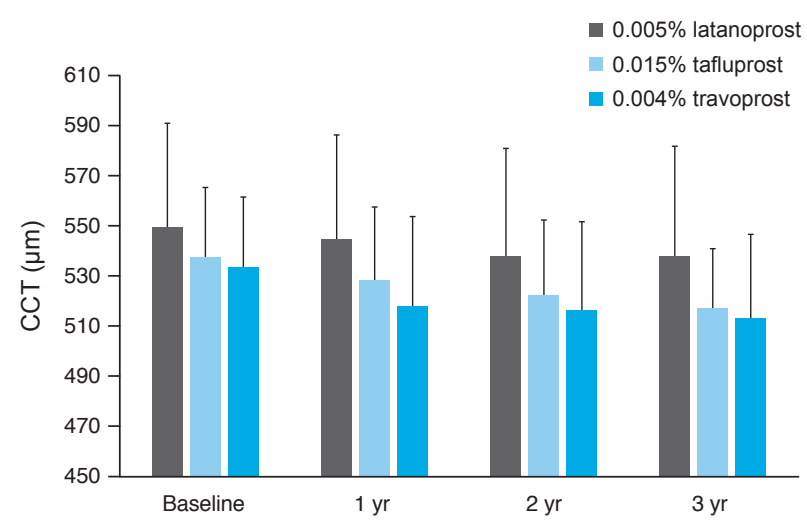

Fig. 2. Mean central corneal thickness (CCT) at baseline and follow-up in the normal tension glaucoma subgroup. The reduction of CCT was confirmed by subanalysis of each subgroup. In the $0.005 \%$ latanoprost group, the mean CCT decreased compared with baseline (baseline, $549.38 \pm 40.02 \mu \mathrm{m}$; 1 year, $544.13 \pm 41.83$ $\mu \mathrm{m}[p=0.11] ; 2$ years, $537.06 \pm 42.64 \mu \mathrm{m}[p=0.00] ; 3$ years, $538.19 \pm 45.44 \mu \mathrm{m}[p=0.02])$. In the $0.0015 \%$ tafluprost group, the mean CCT was significantly decreased compared with baseline (baseline $534.31 \pm 26.85 \mu \mathrm{m}$; 1 year, $523.00 \pm 27.53 \mu \mathrm{m}[p=0.00]$; 2 years, $517.94 \pm 27.93 \mu \mathrm{m}[p=0.00] ; 3$ years, $514.75 \pm 23.22 \mu \mathrm{m}[p$ $=0.00])$. In the $0.004 \%$ travoprost group, the mean CCT was also significantly decreased compared with baseline (baseline, 533.04 $\pm 28.68 \mu \mathrm{m}$; 1 year, $516.78 \pm 37.04 \mu \mathrm{m}[p=0.00]$; 2 years, $513.57 \pm$ $36.05 \mu \mathrm{m}[p=0.00] ; 3$ years, $512.00 \pm 35.15 \mu \mathrm{m}[p=0.00])$. smooth muscle of the ciliary body [20], whereas the corneal effects may be mediated by MMP-2 cross-activation. PGAs might induce corneal thinning by increasing MMP activity. Thus, corneal thinning may progress over a longer follow-up period in PGA patients. Bergonzi et al. [21] suggested that prolonged treatment with PGAs was associated with collagen degradation in the stromal extracellular matrix, owing to activation of MMPs. A long-lasting consequence of this alteration could be CCT reduction.

In this study, we found a statistically significant mean CCT reduction of $17.75 \mu \mathrm{m}$ in NTG patients after 3 years of PGA monotherapy. Several previous studies have reported that use of PGAs for glaucoma treatment reduces CCT [5,14-16]. Viswanathan et al. [22] showed that CCT decreased by a mean of $12.3 \mu \mathrm{m}$ in eyes treated with prostaglandin monotherapy, or with a combination of prostaglandins, over a follow-up period of seven years. Interestingly, mean CCT showed a decreasing trend, and there was a significant difference at 1,2 , and 3 years compared with baseline. The degree of reduction in CCT value was different for each NTG subgroup, but there was no statistical difference among the groups $(p=0.18)$ (Table 3$)$. There have been other studies that have demonstrated similar results [23-25]. Hatanaka et al. [16] reported latanoprost $0.005 \%$, travoprost $0.004 \%$, and bimatoprost $0.03 \%$ were associated with equivalent CCT reductions after eight weeks of treatment, and there was no statistical difference among the groups. Park et al. [26] showed that travoprost and latanoprost decreased mean corneal thickness, but bimatoprost had no effect. It is known that latanoprost and travoprost are activated by hydrolysis in the cornea, while bimatoprost results in relatively little such hydrolysis [27]. Tafluprost is readily hydrolyzed at the corneal surface and converted to the tafluprost acid form to occupy the target prostaglandin F receptors at the site of action [28]. Accordingly, there appears to be no difference in CCT reduction among the three PG formulations compared in this study.

Table 3. Central corneal thickness: baseline and after 3 years of treatment

\begin{tabular}{lccc}
\hline & Baseline & 3 Years & $p$-value \\
\hline $0.005 \%$ latanoprost & $549.38 \pm 40.02$ & $538.19 \pm 45.44$ & $0.02^{*}$ \\
$0.0015 \%$ tafluprost & $534.31 \pm 26.85$ & $514.75 \pm 23.22$ & $0.00^{*}$ \\
$0.004 \%$ travoprost & $533.04 \pm 28.68$ & $512.00 \pm 35.15$ & $0.00^{*}$ \\
\hline
\end{tabular}

There was no statistical difference between groups $(p=0.18)$. Analysis of covariance test was performed.

*Paired-sample $t$-test was performed. 
CCT is known to affect measurement of IOP by the Goldmann applanation test $[10,11]$. Some studies have demonstrated that higher CCT values are related to higher IOP levels and thinner corneas, leading to underestimation of IOP [29-31]. For this reason, we analyzed the correlation between CCT and IOP in each NTG subgroup. At baseline, the correlation between IOP and CCT was as follows: $0.005 \%$ latanoprost $(\mathrm{r}=0.51, p=0.04), 0.0015 \%$ tafluprost ( $\mathrm{r}$ $=-0.08, p=0.78)$, and $0.004 \%$ travoprost $(\mathrm{r}=0.13, p=0.56)$. No significant correlation was apparent between 3-year IOP and CCT values: $0.005 \%$ latanoprost $(\mathrm{r}=0.25, p=$ 0.35 ), $0.0015 \%$ tafluprost ( $\mathrm{r}=0.26, p=0.32$ ), and $0.004 \%$ travoprost $(\mathrm{r}=0.20, p=0.37)$. This suggests that the correlation analysis was limited due to the small number of patients in each group. Moreover, without applying Ehler's IOP correction formula, the correlation between CCT and IOP within each PG analogue group did not seem to yield meaningful results, especially at baseline.

In this study, it is likely that the small sample size is in violation of Ehler's IOP correction formula [10]. However, as in previous studies [4,5,29], lifelong use of PGAs might increase the possibility of overestimating the IOP-lowering effect as CCT thinning progresses. Therefore, measurement of IOP and CCT could help in proper interpretation of IOP.

This study was limited by the nature of retrospective research, a small number of enrolled patients, and a relatively short observation period; further, we did not demonstrate any correlation between IOP and CCT.

We compared CCT between the NTG group with PG analogues and glaucoma suspect group. This method cannot prove that NTG itself reduces CCT as the disease progresses. However, the study is meaningful as there are few papers comparing the mean CCT reduction between PGA subgroups.

In conclusion, topical therapy with PGAs appears to cause a significant decrease in CCT, but there is a difference among NTG subgroups. Long-term follow-up studies including more participants are needed.

\section{Conflict of Interest}

No potential conflict of interest relevant to this article was reported.

\section{References}

1. Coleman AL. Glaucoma. Lancet 1999;354:1803-10.

2. Quigley HA. Number of people with glaucoma worldwide. Br J Ophthalmol 1996;80:389-93.

3. Shah R, Wormald RP. Glaucoma. BMJ Clin Evid 2011;2011: 0703.

4. Kim CS, Seong GJ, Lee NH, et al. Prevalence of primary open-angle glaucoma in central South Korea the Namil study. Ophthalmology 2011;118:1024-30.

5. You JY, Cho BJ. Effect of latanoprost on central corneal thickness in unilateral normal-tension glaucoma. $J$ Ocul Pharmacol Ther 2013;29:335-8.

6. Russo A, Riva I, Pizzolante T, et al. Latanoprost ophthalmic solution in the treatment of open angle glaucoma or raised intraocular pressure: a review. Clin Ophthalmol 2008;2:897-905.

7. Schachtschabel U, Lindsey JD, Weinreb RN. The mechanism of action of prostaglandins on uveoscleral outflow. Curr Opin Ophthalmol 2000;11:112-5.

8. Ooi YH, Oh DJ, Rhee DJ. Effect of bimatoprost, latanoprost, and unoprostone on matrix metalloproteinases and their inhibitors in human ciliary body smooth muscle cells. Invest Ophthalmol Vis Sci 2009;50:5259-65.

9. BenEzra D, Foidart JM. Collagens and non collagenous proteins in the human eye. I. Corneal stroma in vivo and keratocyte production in vitro. Curr Eye Res 1981;1:101-10.

10. Ehlers N, Bramsen T, Sperling S. Applanation tonometry and central corneal thickness. Acta Ophthalmol (Copenh) 1975;53:34-43.

11. Goldmann H, Schmidt T. Applanation tonometry. Ophthalmologica 1957;134:221-42.

12. Alm A, Stjernschantz J. Effects on intraocular pressure and side effects of $0.005 \%$ latanoprost applied once daily, evening or morning: a comparison with timolol. Scandinavian Latanoprost Study Group. Ophthalmology 1995;102:1743-52.

13. Gordon MO, Beiser JA, Brandt JD, et al. The Ocular Hypertension Treatment Study: baseline factors that predict the onset of primary open-angle glaucoma. Arch Ophthalmol 2002;120:714-20.

14. Kim HJ, Cho BJ. Long-term effect of latanoprost on central corneal thickness in normal tension glaucoma. $J$ Ocul Pharmacol Ther 2011;27:73-6.

15. Lee H, Cho BJ. Long-term effect of latanoprost on central corneal thickness in normal-tension glaucoma: five-year follow-up results. J Ocul Pharmacol Ther 2015;31:152-5. 
16. Hatanaka M, Vessani RM, Elias IR, et al. The effect of prostaglandin analogs and prostamide on central corneal thickness. J Ocul Pharmacol Ther 2009;25:51-3.

17. Greve EL, Rulo AH, Drance SM, et al. Reduced intraocular pressure and increased ocular perfusion pressure in normal tension glaucoma: a review of short-term studies with three dose regimens of latanoprost treatment. Surv Ophthalmol 1997;41 Suppl 2:S89-92.

18. McKibbin M, Menage MJ. The effect of once-daily latanoprost on intraocular pressure and pulsatile ocular blood flow in normal tension glaucoma. Eye (Lond) 1999;13(Pt 1):31-4.

19. Sivak JM, Fini ME. MMPs in the eye: emerging roles for matrix metalloproteinases in ocular physiology. Prog Retin Eye Res 2002;21:1-14.

20. Wang N, Lindsey JD, Angert M, Weinreb RN. Latanoprost and matrix metalloproteinase-1 in human choroid organ cultures. Curr Eye Res 2001;22:198-207.

21. Bergonzi C, Giani A, Blini M, et al. Evaluation of prostaglandin analogue effects on corneal keratocyte density using scanning laser confocal microscopy. J Glaucoma 2010;19:617-21.

22. Viswanathan D, Goldberg I, Graham SL. Longitudinal effect of topical antiglaucoma medications on central corneal thickness. Clin Exp Ophthalmol 2013;41:348-54.

23. Maruyama Y, Mori K, Ikeda Y, et al. Effects of long-term topical prostaglandin therapy on central corneal thickness. J Ocul Pharmacol Ther 2014;30:440-4.
24. Stefan C, Dumitrica DM, Tebeanu E, et al. Prostaglandin analogues and central corneal thickness. Oftalmologia 2007;51:95-9.

25. Zhong Y, Shen X, Yu J, et al. The comparison of the effects of latanoprost, travoprost, and bimatoprost on central corneal thickness. Cornea 2011;30:861-4.

26. Park MH, Cho K, Moon JI. The effects of prostaglandin analogues on the corneal thickness. $J$ Korean Ophthalmol Soc 2009;50:565-71.

27. Maxey KM, Johnson JL, LaBrecque J. The hydrolysis of bimatoprost in corneal tissue generates a potent prostanoid FP receptor agonist. Surv Ophthalmol 2002;47 Suppl 1:S3440.

28. Fukano Y, Kawazu K, Akaishi T, et al. Metabolism and ocular tissue distribution of an antiglaucoma prostanoid, tafluprost, after ocular instillation to monkeys. J Ocul Pharmacol Ther 2011;27:251-9.

29. Doughty MJ, Zaman ML. Human corneal thickness and its impact on intraocular pressure measures: a review and meta-analysis approach. Surv Ophthalmol 2000;44:367-408.

30. Wu LL, Suzuki Y, Ideta R, Araie M. Central corneal thickness of normal tension glaucoma patients in Japan. Jpn J Ophthalmol 2000;44:643-7.

31. Singh RP, Goldberg I, Graham SL, et al. Central corneal thickness, tonometry, and ocular dimensions in glaucoma and ocular hypertension. J Glaucoma 2001;10:206-10. 